\title{
Research on a kind of laser beam side axis positioning technology in space
}

\author{
Ziqiang Hao
}

Changchun University of Science and Technology, Changchun, Jilin, 130022 China

Keywords: laser positioning; aerosol scattering; scattering angel.

\begin{abstract}
Since the laser beam divergence angle is very small, and there is no scattering effect in the space, the positioning technology of laser source is a difficult problem in the field of laser detection and interference. In this paper, a new method based on the scattering of the laser beam is presented, by using MIE scattering theory the theoretical analysis and numerical calculation of the properties of the light scattering of particles is carried out. The relationship between the scattering light distribution and the size parameters of the microspheres and the complex refractive index is obtained. The results show that the scattering intensity of the aerosol particles is mainly distributed in the forward direction, and the scattering angle decreases with the increase of the scattering angle. It can be concluded that the laser beam based on aerosol scattering is feasible.
\end{abstract}

\section{Introduction}

With the development of laser technology, laser communication has become the main research direction of communication technology. And the detection and interference of laser communication has become one of the main research contents in the military field. However, because of the small laser beam divergence angle, no scattering effect in the space environment, so the detection technology of laser communication becomes to be a difficult problem. The detection of the weak signal by side axis positioning technology has the important significance not only in the field of laser communication, but also in the field of Laser Guidance Countermeasure. At present, the technology of laser detection a is still used by direct detection. It requires a large number of laser detection equipments, low efficiency, high cost, difficult operation, and it is not suitable for space communications laser capture and positioning.

\section{Theoretical Model}

When the laser propagation in the space, the scattering effect is very tiny. So we should mainly considerate the aerosol particles scattering effect. It includes not only the scattering effect, but also the absorption effect of aerosol particles in the model. Set of the radius of aerosol particles $r 1-r 2$, the distribution function of aerosol particles is $n(r)$, the laser beam is ideal light.

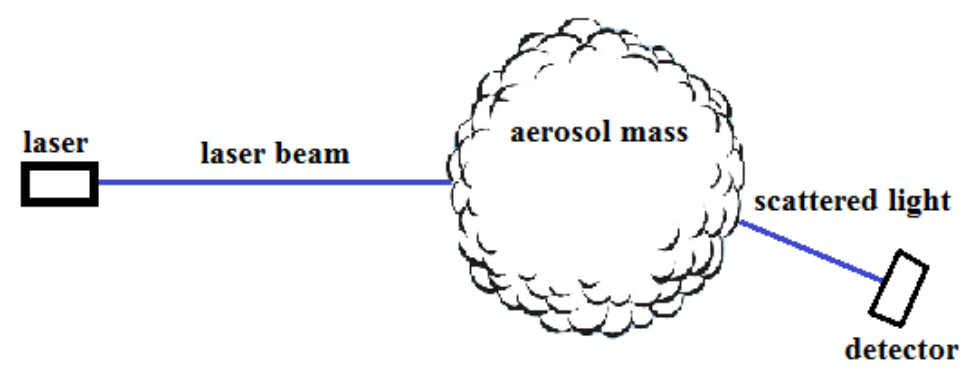

Fig.1 Side axis positioning model

For the Mie scattering theory, when the incident light is non-polarized light, the scattered light intensity and attenuation factor of aerosol particles can be obtained:

$\mathrm{I}_{\text {sca }}=\lambda^{2} \mathrm{I}_{0}\left(|\mathrm{~s} 1(\theta)|^{2}+|\mathrm{s} 2(\theta)|^{2}\right) / 2 \mathrm{k}^{2} \mathrm{~L}^{2}(1)$
$\mathrm{Q}_{\text {ext }}=2 / \mathrm{x}^{2} \sum^{\infty}(2 \mathrm{n}+1) \operatorname{Re}\left(\mathrm{a}_{\mathrm{n}}+\mathrm{b}_{\mathrm{n}}\right)(2)$

In formula (1),L is the distance of the observation point to the center of the sphere of scattering particles, $\lambda$ is the wavelength of the incident light wave, $\theta$ is the scattering angle, amplitude 
function $s 1(\theta)$, s2 $(\theta)$ Mie coefficient $a_{n}, b_{n}$, the calculation formulas of scattering angle function $\pi_{n}(\cos \theta), \tau_{n}(\cos \theta)$ are as follows:

$$
\begin{aligned}
& \mathrm{s} 1(\theta)=\sum_{\mathrm{n}=1}^{\infty} \frac{2 \mathrm{n}+1}{\mathrm{n}(\mathrm{n}+1)}\left(\mathrm{a}_{\mathrm{n}} \pi_{\mathrm{n}}(\cos \theta)+\mathrm{b}_{\mathrm{n}} \tau_{\mathrm{n}}(\cos \theta)\right)(3) \\
& \operatorname{s} 2(\theta)=\sum_{n=1}^{\infty} \frac{2 n+1}{n(n+1)}\left(a_{n} \tau_{n}(\cos \theta)+b_{n} \pi_{n}(\cos \theta)\right)(4) \\
& \mathrm{a}_{\mathrm{n}}=\frac{\Psi_{\mathrm{n}}(\mathrm{x}) \Psi_{\mathrm{n}}^{\prime}(\mathrm{mx})-\mathrm{m} \Psi_{\mathrm{n}}^{\prime}(\mathrm{x}) \Psi_{\mathrm{n}}(\mathrm{mx})}{\xi_{\mathrm{n}}(\mathrm{x}) \Psi_{\mathrm{n}}^{\prime}(\mathrm{mx})-\mathrm{m} \xi_{\mathrm{n}}^{\prime}(\mathrm{x}) \Psi_{\mathrm{n}}(\mathrm{mx})}(5) \\
& b_{n}=\frac{m \Psi_{n}(x) \Psi_{n}^{\prime}(m x)-\Psi_{n}^{\prime}(x) \Psi_{n}(m x)}{m \xi_{n}(x) \Psi_{n}^{\prime}(m x)-\xi_{n}^{\prime}(x) \Psi_{n}(m x)}(6) \\
& \pi_{\mathrm{n}}(\cos \theta)=\mathrm{P}_{\mathrm{n}}^{1}(\cos \theta) / \sin \theta(7) \\
& \tau_{\mathrm{n}}(\cos \theta)=\frac{\mathrm{d}}{\mathrm{d} \theta} \mathrm{P}_{\mathrm{n}}^{1}(\cos \theta)(8) \\
& \Psi_{\mathrm{n}}(\mathrm{x})=\left(\frac{\pi_{\mathrm{x}}}{2}\right)^{\frac{1}{2}} \mathrm{~J}_{\mathrm{n}+\frac{1}{2}(\mathrm{x})} \quad \xi_{\mathrm{n}}(\mathrm{x})=\frac{\pi_{\mathrm{n}}{ }^{\frac{1}{2}}}{2} \mathrm{H}_{\mathrm{n}+\frac{1}{2}(\mathrm{x})}
\end{aligned}
$$

$\mathrm{J}_{\mathrm{n}+\frac{1}{2}(\mathrm{x})}$ is the half-integer-order Bessel function, $\mathrm{H}_{\mathrm{n}+\frac{1}{2}(\mathrm{x})}$ is the first type Hankel function of half-integer order, $\mathrm{P}_{\mathrm{n}}^{1}(\cos \theta)$ is 1 order $\mathrm{n}$ times associated Legendre function, scale parameter (dimensionless particle size) $\mathrm{x}=2 \pi_{\mathrm{r}} / \lambda, \mathrm{r}$ is the radius of aerosol particles , $\mathrm{m}$ is the refractive index of the scattering particles relatives the surrounding medium.

According to the scattering intensity distribution function,it can be obtained the body angle scattering coefficient:

$$
\beta_{\mathrm{pj}}(\theta, \lambda)=\frac{\lambda^{2}}{4 \pi^{2}} \int_{\mathrm{r} 1}^{\mathrm{r} 2} \mathrm{n}(\mathrm{r})\left|\mathrm{s}_{\mathrm{j}}(\theta)\right|^{2} \mathrm{dr}(9)
$$

$\mathrm{n}(\mathrm{r})$ is a function of the particle scale, $\mathrm{r} 1, \mathrm{r} 2$ are the upper and lower scale of particle, $\left|s_{j}(\theta)\right|^{2}$ is the intensities distribution function of the two polarization directions. Scattering intensity can be characterized by the body angle scattering coefficient:

$\mathrm{I}(\theta)=\mathrm{I}_{0} \beta(\theta) / \mathrm{L}^{2}(10)$

$\mathrm{I}_{0}$ is the first incident light intensity, $\mathrm{L}$ is distance from observation point to the scattering center, When the incident light is unpolarized light:

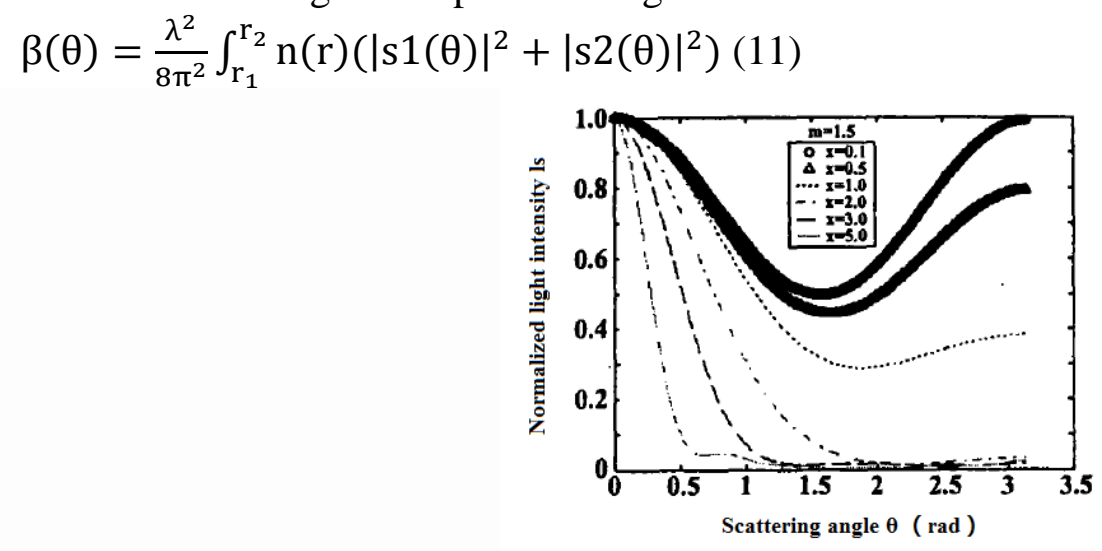

Fig. 2 Scatter diagrams of different scale parameters

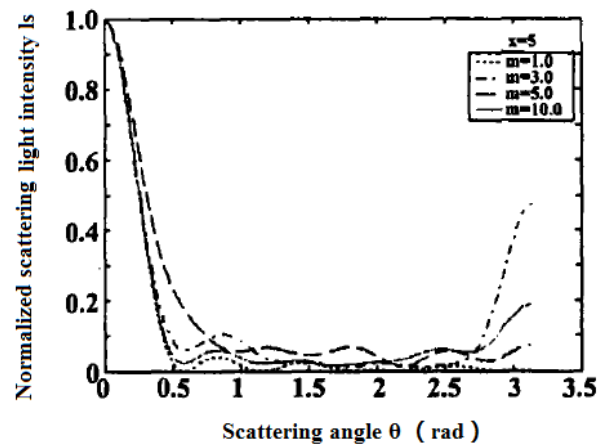

Fig. 3 Scatter diagrams of same scale parameters different complex index of refraction 


\section{Numerical calculation and analysis}

Based on the formula (1) (8), using MATLAB language to write the corresponding program, it can be calculated the numerical results of the scattered light intensity distribution. Rescaling parameter $\mathrm{x}$, the relative refractive index $\mathrm{m}$, it can be drawn the relationship of the distribution of scattered light intensity $\mathrm{I}_{\text {sca }}$ with Parameters $\mathrm{x}$ and $\mathrm{m}$. Fig.2 shows the distribution of particle scattering into regular changes, when the particles scale parameter is small, scattering intensity distributes closed to the Rayleigh scattering. With the increase of the scale parameter, the forward scattered light intensity is getting stronger, backscatter decreased significantly. In the range of small angle forward, As the angle increases, scattered light intensity decline sharply, thus from a single particle scattering angle, it offers the possibility of using forward scattered light intensity change to measure the scattering angle. Fig. 3 has little effect on the refractive index of the forward scattering intensity, when refractive indexm $=0.3$, Backscatter strength has a clear peak.

To single dispersion, uniform scale particle-based, obtained by the formula(11), body angle scattering coefficient is proportional to the average intensity distribution function of the two polarization directions; for more decentralized system, the key of solving body angle scattering coefficient is to solve the particle size distribution function, here the particle size distribution function takes the lognormal spectrum closest to the actual state of the atmosphere:

$$
\mathrm{n}(\mathrm{r})=\frac{\mathrm{dN}}{\mathrm{d} \ln \mathrm{r}}=\frac{\mathrm{N}}{\sqrt{2 \pi} \ln \sigma} \exp \left[\frac{\left(\operatorname{lnr}-\operatorname{lnr}_{\mathrm{bar}}\right)^{2}}{2(\ln \sigma)^{2}}\right](12)
$$

$\mathrm{r}_{\mathrm{bar}}$ is the mean radius of geometric, $\sigma$ is the standard deviation of geometric, $\mathrm{N}$ is the number of particles of per unit volume.

Fig.4 gives the size distribution function of the typical aerosol model. Fig. 5 shows the corresponding body angle scattering coefficients. During calculating the body angle scattering coefficients, take laser wavelength $1.06 \mu \mathrm{m}$, the complex refractive index of the selected reference to its relationship with atmospheric humidity. Humidity was $87 \%$ and $66 \%$, to the complex refractive index corresponded $m=1$. 33,1. 36.It can be seen that scattered light intensity focused on forward from Fig. 5, and as the scattering angle increases, the scattered light intensity drops . Therefore, it can be taken two scatters A,B within forward scattering as Fig. 1, scattering angles are $\theta, \theta+\omega$, a planar array detector parallel a data acquisition card, mainly records the time of detector receives scattered light from individual scatters. the time difference of scattered light from A and B is $\mathrm{t}$, according to formula(2),(10), plane array detectors can be measured the light intensity of $A$ and $B$ :

$$
\begin{aligned}
& \mathrm{I}_{\text {Asca }}=\mathrm{I}_{\mathrm{A}} \beta(\theta) \exp \left(-\mathrm{Q}_{\text {ext }} \mathrm{L}_{\mathrm{A}}\right) / \mathrm{L}_{\mathrm{A}}^{2}(13) \\
& \mathrm{I}_{\mathrm{Bsca}}=\mathrm{I}_{\mathrm{B}} \beta(\theta+\omega) \exp \left(-\mathrm{Q}_{\mathrm{ext}} \mathrm{L}_{\mathrm{B}}\right) / \mathrm{L}_{\mathrm{B}}^{2}(14) \\
& \mathrm{I}_{\mathrm{B}}=\mathrm{I}_{\mathrm{A}} \exp \left(-\mathrm{Q}_{\text {ext }} \mathrm{L}_{\mathrm{AB}}\right), \frac{\mathrm{L}_{\mathrm{A}}}{\mathrm{L}_{\mathrm{B}}}=\frac{\sin (\theta+\omega)}{\sin \theta}, \mathrm{L}_{\mathrm{B}}+\mathrm{L}_{\mathrm{AB}}-\mathrm{L}_{\mathrm{A}}=\mathrm{ct}, \quad \mathrm{c} \text { is the speed of light, } \mathrm{f}(\theta)= \\
& \mathrm{I}_{\text {Asca }} / \mathrm{I}_{\text {Bsca }} \text {, you can get: } \\
& f(\theta)=\frac{I_{\text {Asca }}}{I_{\text {Bsca }}}=\frac{\beta(\theta) \sin ^{2} \theta \exp \left(Q_{\text {exp }} \mathrm{ct}\right)}{\beta(\theta+\omega) \sin ^{2}(\theta+\omega)}(15)
\end{aligned}
$$

$\theta$ can be obtained from formula(15), the location information of the laser beam can be determined according to the model geometry.

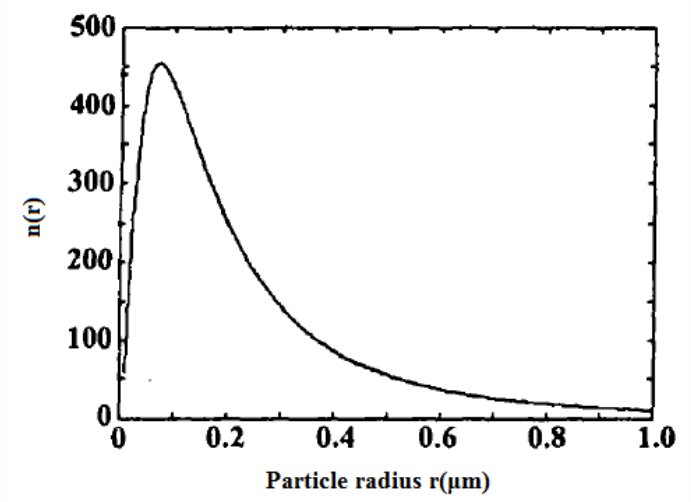

Fig. 4 Distribution of aerosol particle radius 


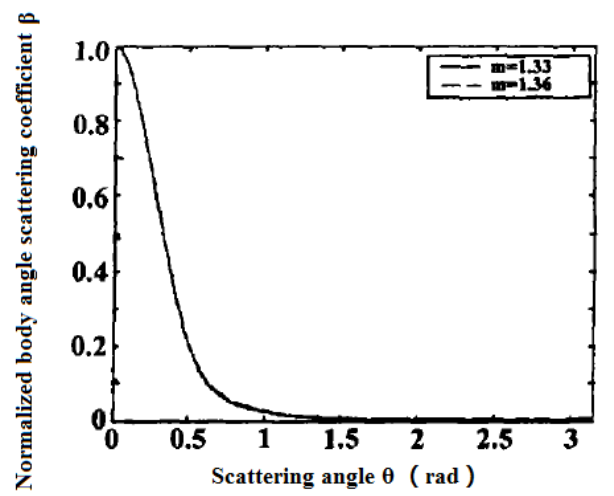

Fig. 5 Normalized body angle scattering coefficient

\section{Conclusion}

This paper analysis the typical aerosol particles scattering characteristics theoretically using Mie scattering theory. From the numerical results, with the scattering particle scale parameter $\mathrm{x}$ increases, scattered light intensity increases concentrated to the forward scattering ; The former condition has little effect to the complex refractive index of the particle to scattering light. This paper calculates and analysis the aerosol body angle scattering coefficient of typical aerosol particles, and analysis the feasibility of using the relationship between body angle scattering coefficient and the scattering angle to achieve side axis positioning of the laser beam.

\section{Acknowledgement}

This work is supported by project of Jilin Provincial Education Department, Youth Fund Project of CUST.

\section{Reference}

[1]Jean - Pierre Cariou. off - axis detection of pulsed laser beams: simulation and measurements in the lower at mosphere [ J] . Proceeding of SP IE Vol. 5086 ( 2003) : 129- 138.

[2]V. Grasso, F. Neri, E. Fucile. Simple angle- resolved light scattering photometer using a photodiode array [ J] . SPIE Vol.2506: 763- 772.

[5]Sylvain Lecler, Yoshitate Takakura , Patrick Meyrueis. Properties of a three- dimensional photonic jet [ J] . Optics Letters, 2005, Vol.30, No. 19. 